\title{
Solitary keratoacanthoma involving upper lip: a diagnostic dilemma - case report and a brief review
}

\author{
Prashant Bhimrao Patil ${ }^{1}$, Vanita Rathor ${ }^{2}$, Sreenivasan Venkatraman ${ }^{3}$, Susmita Saxen ${ }^{4}$, Nagaraju Kamarthi ${ }^{5}$
}

\author{
${ }^{1}$ MDS, Professor, Department of Oral Medicine and Radiology. \\ ${ }^{2}$ MDS, Reader, Department of Oral Pathology and Microbiology. \\ ${ }^{3}$ MDS, Professor and Head Department of Oral Medicine and Radiology. \\ ${ }^{4}$ MDS, Professor and Head Department of Oral pathology and Microbiology. \\ ${ }^{5}$ MDS, Senior Lecturer, Department of Oral Medicine and Radiology.
}

\section{Correspondence:}

Dr. Prashant B Patil MDS

Professor, Department of Oral Medicine and Radiology

Subharti Dental College, $\mathrm{NH}-58$, Subhartipuram,

Meerut, Uttar Pradesh, India-250 002,

Email:prashantomdr@gmail.com.

\begin{abstract}
Keratoacanthoma (KA) is a benign epithelial proliferative lesion which frequently occurs on the sun exposed areas of the skin. KA originates within the pilosebaceous apparatus of the skin and may be solitary or multiple. Solitary KA can be difficult to differentiate from squamous cell carcinoma (SCC) both clinically and microscopically. However, the clinical course of the lesion and its ability to self involute makes it a distinct clinical entity. Solitary KA appears on the vermilion border of the lower lips with some frequency. A case of keratoacanthoma involving the upper lip is reported presenting as an exophytic growth that resolved after excisional biopsy. Photographic documentation of the case along with relevant management protocol is discussed. The article emphasizes the significance of recognizing such lesion and discriminating it from SCC thus carrying diagnostic and therapeutic implications. However, in case of dilemma it is prudent to assume that the lesion is SCC unless proved otherwise clinically or histologically.
\end{abstract}

Key words: Keratoacanthoma, squamous cell carcinoma, upper lip, self involution, regression after biopsy. 


\section{Introduction}

Keratoacanthoma is defined as a benign and usually rapidly growing epithelial proliferation originating in the supra sebo-glandular portion of hair follicles (1-3). Lesions typically present as a solitary, firm, skin colored reddish papules that rapidly progress to dome shaped sessile, nodule with a central crateriform ulceration or keratin plug.

The lesion shows a male preponderance and occurs frequently on sun exposed areas of face, neck, forearms and in older age group $(2,4)$. KA can appear frequently on the vermilion border of lips thus mandating the dental professionals to be aware of the lesion. Etiology of KA still remains obscure; however actinic rays, HPV, trauma, genetic factors and immunocompromised status have been implicated (2-6). Solitary KA is often difficult to differentiate from well-differentiated SCC both clinically and histologically. The difference in diagnosis between these two lesions is of paramount importance since it carries therapeutic implications, thus prompting us to report this case. The case is unique in that $\mathrm{KA}$ is reported in a young individual involving upper lip contrary to the previous reported cases occurring on the lower lip and in older age group usually above 40 years of age (1-3, 5-8).

\section{Case Report}

A 26 year old male was referred from Department of Dermatology to the outpatient Department of Oral Medicine and Radiology for evaluation of an exophytic growth on the upper lip with one and half years of duration. There was no associated history of previous local trauma, but the patient recalls occurrence of acute onset ulcers involving mouth and lips before one and half years which subsided following local therapy. The lip lesion did not resolve and persisted with intermittent variation in the size. Patient was a smoker since 6-7 years and occasional gutkha chewer. Facial examination revealed well-demarcated sessile exophytic nodular growth located at the junction of vermilion border and the skin of upper lip (Fig.1). The lesion was non-tender, firm with no discharge. Palpation of neck did not disclose any suspicious lymph nodes and medical history was negative. An initial differential hypothesis of actinic cheilosis, keratoacanthoma, squamous cell carcinoma, bacterial or mycotic infection was made and the patient was subjected for complete hemogram along with $\mathrm{x}$-ray chest and Mantoux test. The test results showed no admissible changes. The need for biopsy was discussed with the patient but could not meet his approval.

Patient reported back after 2 months from his initial visit with no change in the size of the lesion, however, the lesion exhibited a yellowish white central zone of plug which was likely to be keratinous mass with raised edges. Subsequently the lesion was completely excised

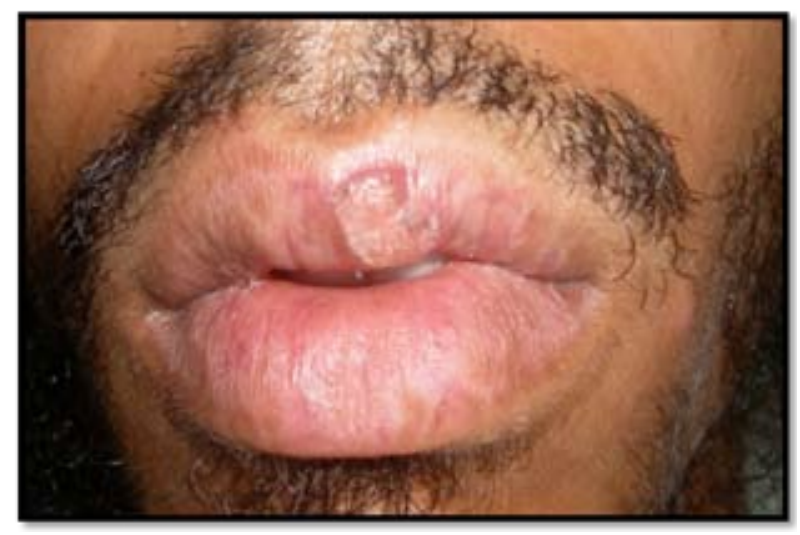

Fig. 1. Clinical photograph showing well-demarcated exophytic nodular growth located at the junction of vermilion border and the skin of upper lip.

with a wedge excision followed by primary closure and the tissue was sent for histopathologic examination. The histopathology report showed proliferating parakeratinized stratified squamous epithelium with thickened stratum granulosum and slightly proliferative spinous and basal cell layers. At numerous places, epithelium showing downward growth forming budding islands with central portion of the island showing keratin (Fig.2 and 3). These down growth islands were surrounded by intense chronic inflammatory infiltrate predominantly comprising of lymphocytes, histiocytes and macrophages. The pathological findings were considered compatible with keratoacanthoma. The patient reported seven days after the biopsy with favorable healing observed. Association of this observation with clinical course of the lesion coupled with the histopathological presentation confirmed the hypothesis of keratoacanthoma. The patient was regularly followed up at every two weeks of interval. There was no recurrence of the lesion after 6 months of follow up.

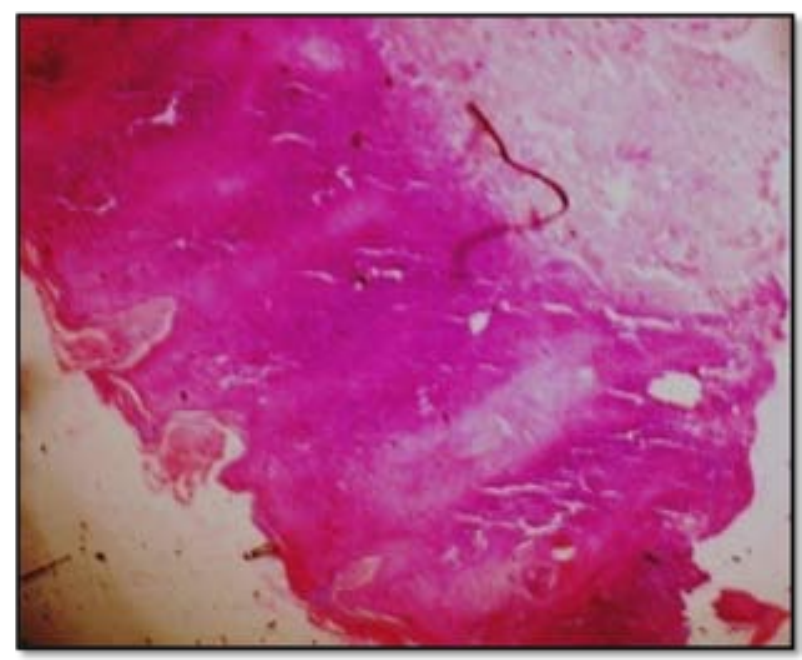

Fig.2. Photomicrograph - (4x) showing hyperplastic stratified squamous epithelium covered by thick layer of parakeratin with central plugging. 


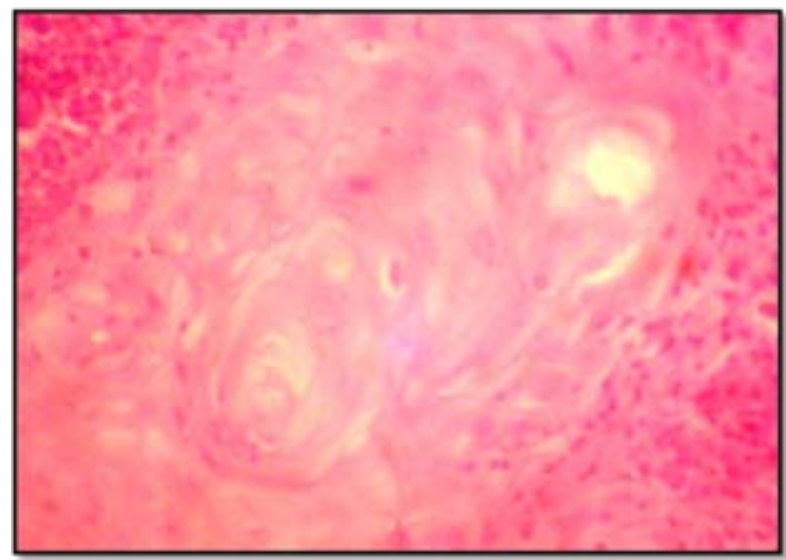

Fig. 3. Photomicrograph (40x) showing epithelial island projecting towards connective tissue with ballooning cell filled with keratin.

\section{Discussion}

$\mathrm{KA}$ is a well known benign squamous epithelial neoplasm with a strong clinical and histological similarity to well-differentiated $\operatorname{SCC}(2,3,5,6)$. Few authors are of the opinion that solitary KA represents an extremely low grade $\operatorname{SCC}(5,6)$. If so it fails to explain the static or regressive behavior of KA. In an attempt to discriminate the two lesions a comparative chromosomal aberration analysis study by Clausen et al. (9) showed that chromosomal instability was significantly higher in cutaneous SCC than in KAs. This difference in genomic expressivity of the two lesions has been correlated to their different biological behavior and pathogenicity. Irritation in the form of actinic rays, trauma, tars, virus which are common implications for both KA and SCC, the difference in the type of tumor produced was explained by experimental work by Ghadially (10) showing that the timing when the irritant took effect whether in active phase or inactive phase was determinant. KAs are thus perverted products of hair follicles with some kind of force still controlling the growth and/or regressive behavior of the lesion. Further the author is of the opinion that KA may persist if the exposure to the irritant is prolonged or in some manner there is inhibition of the normal cyclic influences on the tissue of origin. Thus SCC is considered as a biologically malignant neoplasm with the ability to metastasize and invade in contrast to KA which undergoes spontaneous resolution and hence considered a self limiting and benign tumor.

Occurrence of solitary KA on vermilion portion of lips is now widely recognized with a reported frequency of $12 \%$ (2). KA of the lip may arise from skin rather than labial mucosa (3), which could well have been the case in the present patient. Though the patient correlates the lesion on lip with occurrence of oral ulcers which healed with local therapy with no episode of reoccurrence, we assume that this part of history is red - herring and it was merely a coincidence. The clinical course of KA is usually typical of the lesion. It begins as nodule grows rapidly in size over a period of 4-5 weeks, remains static for another 4-8 weeks before undergoing spontaneous involution with the expulsion of keratin and complete resolution observed in the next 6-8 weeks period of time (4). Cases of solitary KA have been reported to persist for a year or more before undergoing spontaneous resolution $(2,4)$. As in the present case the lesion persisted for almost one and half year before showing complete resolution after surgical intervention. This unusual clinical course of KA has been the subject of considerable conjecture thus evoking considerable debate regarding the treatment approach. Some authors are of the opinion that a wait and watch policy should be adapted for KA thus allowing spontaneous regression without any intervention $(7,11)$. It is hypothesized that by some unexplained immunological mechanism and/or the behavior of the parent tissue of origin could be the possible reasons for self resolution of KA (2). A similar example of spontaneous resolution has been described with other lesions such as spitz tumor (12), pilocytic astrocytoma (13). Few other authors recommend surgical intervention for solitary KA as every case does not show self involution and further self resolution often leaves a depressed pigmented scar which can be unaesthetic (2).

As stated above apart from observational stance and surgical excision various other treatment modalities have been proposed including cryosurgery, lasers, curettage and cauterization, radiotherapy, topical podophyllin and 5-flourouracil, intralesional bismuth, bleomycin, interferon alfa- $2 \mathrm{a}$, mehtotrexate and triamcinolone (4-6). But most of them seem to be too variegated and have any effect on the final outcome of the disease. Visscher et al. (3) reported a case of solitary giant keratoacanthoma treated with intralesional methotrexate with good prognosis. Solitary lesions approximating $1-1.5 \mathrm{~cm}$ have been traditionally excised with a wedge excision $(3,5,6)$. Such an approach has the advantage of obtaining entire lesion for histological examination and also the resulting scar is usually less disfiguring than the scar remaining after self involution of the lesion. In our case the patient recalls the static or regressive behavior of the lesion more suggestive of KA than a true neoplasm. Further, complete healing was observed after excisional biopsy which is unusual to be seen in a true neoplasm. Either spontaneous regression or healing by surgical intervention is considered as definitive evidence of KA (7). Thus the present case documents complete resolution of the lesion after surgical intervention indicative of KA. It is thus rationale to include KA in the diagnostic hypothesis list of every solitary nodular or ulcerative condition of lip. Further studies may be required to better understand the mechanisms involved in tumor proliferation, growth controlling factors, and involution of KA.

\section{References} e36 
on an unusual lesion. Can Med Assoc J. 1965;93:272-3.

2. Hardman FG.Keratoacanthoma on the lips. Br J Oral Surg. 1971;9:46-53.

3. de Visscher JG, van der Wal JE, Starink TM, Tiwari RM, van der Waal I. Giant keratoacanthoma of the lower lip. Report of a case of spontaneous regression. Oral Surg Oral Med Oral Pathol Oral Radiol Endod. 1996;81:193-6.

4. Schwartz RA. Keratoacanthoma. J Am Acad Dermatol. 1994;30:119; quiz 20-2.

5. de Visscher JG, van der Wal KG, Blanken R, Willemse F. Treatment of giant keratoacanthoma of the skin of the lower lip with intralesional methotrexate: a case report. J Oral Maxillofac Surg. 2002;60:93-5.

6. Karnauchow PN. A lesion simulating squamous carcinoma of the lip (keratoacanthoma). Can Med Assoc J. 1958;78:346-8.

7. Ramos LM, Cardoso SV, Loyola AM, Rocha MA, Durighetto-Júnior AF. Keratoacanthoma of the inferior lip: review and report of case with spontaneous regression.J Appl Oral Sci. 2009;17:262-5.

8. Eversole LR, Leider AS, Alexander G. Intraoral and labial keratoacanthoma. Oral Surg Oral Med Oral Pathol. 1982;54:663-7.

9. Clausen OP, Aass HC, Beigi M, Purdie KJ, Proby CM, Brown VL, et al. Are keratoacanthomas variants of squamous cell carcinomas? A comparison of chromosomal aberrations by comparative genomic hybridization. J Invest Dermatol. 2006;126:2308-15.

10. Ghadially FN. Comparative morphological study of the keratoacanthoma of man and similar experimentally produced lesions in the rabbit. J Path Bact. 1958;75:441.

11. Griffiths RW. Keratoacanthoma observed. Br J Plast Surg. 2004;57:485-501.

12. Zalaudek I, Bonifazi E, Ferrara G, Argenziano G. Keratoacanthomas and spitz tumors: are they both 'self-limiting' variants of malignant cutaneous neoplasms? Dermatology. 2009;219:3-6.

13. Gallucci M, Catalucci A, Scheithauer BW, Forbes GS. Spontaneous involution of pilocytic astrocytoma in a patient without neurofibromatosis type 1: case report. Radiology. 2000;214:223-6. 